\title{
On Rationally Supported Surfaces
}

\author{
Jens Gravesen ${ }^{a}$, Bert Jüttler ${ }^{b}$, and Zbyněk Š́rir ${ }^{c}$ \\ ${ }^{a}$ Technical University of Denmark, Department of Mathematics, Kgs. Lyngby \\ ${ }^{b}$ Johannes Kepler University, Institute of Applied Geometry, Linz, Austria \\ ${ }^{c}$ University of West Bohemia, Faculty of Applied Sciences, Department of \\ Mathematics, Plzeñ, Czech Republic
}

\begin{abstract}
We analyze the class of surfaces which are equipped with rational support functions. Any rational support function can be decomposed into a symmetric (even) and an antisymmetric (odd) part. We analyze certain geometric properties of surfaces with odd and even rational support functions. In particular it is shown that odd rational support functions correspond to those rational surfaces which can be equipped with a linear field of normal vectors, which were discussed by Sampoli et al. (2006). As shown recently, this class of surfaces includes non-developable quadratic triangular Bézier surface patches (Lávička and Bastl, 2007; Peternell and Odehnal, 2007).
\end{abstract}

Key words: rational support function, $L N$-surfaces, triangular quadratic Bézier surface patches.

\section{Introduction}

The support function representation describes a surface as the envelope of its tangent planes, where the distance between the tangent plane and the origin is specified by a function of the unit normal vector. This representation is one of the classical tools in the field of convex geometry (see Bonnesen, 1987; Groemer, 1996; Gruber, 1993). In addition, it provides a computationally simple way to extract curvature information (Gravesen, 2007). Its application to problems from Computer Aided Design dates back to a classical paper of Sabin

Email addresses: j.gravesen@mat.dtu.dk (Jens Gravesen $\left.{ }^{a}\right)$, bert.juettler@jku.at (Bert Jüttler $\left.{ }^{b}\right)$, zbynek.sir@mff.cuni.cz (Zbyněk Šír $\left.{ }^{c}\right)$. 
(1974). In particular, it is promising to apply this representation to issues related to offsets and convolution of surfaces, as these operations correspond to simple algebraic operations of the corresponding support functions.

We consider rational support functions, i.e. functions obtained by restricting a rational function to the unit sphere, which is seen as the set of unit normal vectors. One obtains rational surfaces which are closed under offsetting and under the more general operation of convolutions (see Sampoli et al., 2006).

In order to deal with the arc length of curves and offsets, Farouki and Sakkalis (1990) established the concept of Pythagorean hodograph representations of curves and surfaces, see the survey article by Farouki (2002) and the references cited therein. This notion has been extended to the surface case by Pottmann (1995), who introduced the class of Pythagorean Normal (PN) surfaces. Later, Peternell and Pottmann (1998) studied these surfaces in the frame of Laguerre geometry. The set of PN surfaces is not closed under convolutions.

Rationally supported surfaces are a special case of the more general class of PN surfaces. Among them, surfaces with polynomial support functions play a particular role, since they are related to harmonic analysis on the sphere (see Š́r et al., 200x). As another generalization one may also consider surfaces defined by piecewise polynomial support functions. i.e., by spherical splines, see Alfeld at al. (1996). The case of piecewise linear representations has been studied by Almegaard et al. (2007).

The remainder of the paper is organized as follows. The next section recalls the notion of polarity and introduces the support function representation of surfaces. Section 3 analyzes rational support functions, in particular with respect to their symmetry properties. Sections 4 and 5 discuss surfaces with odd and even rational support functions, respectively. Finally we conclude this paper.

\section{Preliminaries}

After recalling the notion of polarity with respect to the unit sphere, we define the dual and primal hypersurfaces which are associated with a $C^{\infty}$ smooth support functions on the unit sphere.

\subsection{The polarity with respect to the unit sphere}

Throughout this paper, let $S$ denote the unit sphere in $\mathbb{R}^{d}$. The notions of surfaces and planes are used as synonyms for hypersurfaces and hyperplanes, respectively. 
The polarity $\pi$ with respect to $S$ maps points to planes and vice versa with the property that $\pi^{2}=\pi \circ \pi$ is the identity (see e.g. Hoschek, 1983). More precisely, the image of the point

$$
\mathbf{p}=\left(p_{1}, p_{2}, \ldots, p_{d}\right)^{\top}
$$

is the plane

$$
\mathbf{P}=\left\{\mathbf{x}=\left(x_{1}, x_{2}, \ldots, x_{d}\right)^{\top}: 1=\mathbf{p} \cdot \mathbf{x}=p_{1} x_{1}+p_{2} x_{2}+\cdots+p_{d} x_{d}\right\}
$$

and vice versa, i.e., $\mathbf{P}=\pi(\mathbf{p})$ and $\mathbf{p}=\pi(\mathbf{P})$. The plane $\pi(\mathbf{p})$ is perpendicular to the ray $\mathbb{R}_{+} \mathbf{p}$. It intersects the ray at the point with distance $1 /\|\mathbf{p}\|$ to the origin.

The polarity $\pi$ is defined for all points $\mathbf{p} \neq \mathbf{0}=(0, \ldots 0)^{\top}$ and for all planes that have a non-zero distance to the origin. These restrictions can be avoided by considering the projective closure of $\mathbb{R}^{d}$. The polarity $\pi$ preserves the relation of incidence between points and planes: The point $\mathbf{p}$ belongs to a plane $\mathbf{Q}$ if and only if the point $\pi(\mathbf{Q})$ belongs to the plane $\pi(\mathbf{p})$.

\subsection{Support function representation of surfaces}

For any function $h$ defined on the sphere $S$, we define two surfaces, which will be called the dual and the primal surface. Let $U \subset S$ be an open subset of the unit sphere $S$, i.e., the intersection of an open set in $\mathbb{R}^{d}$ with $S$. We consider a given function $h \in \mathcal{C}^{\infty}(U, \mathbb{R})$ with the property that $h(\mathbf{n}) \neq 0$ for $\mathbf{n} \in S$.

The dual surface associated with $h$ is described by the mapping $\mathbf{d}_{h} \in \mathcal{C} \infty\left(U, \mathbb{R}^{d}\right)$,

$$
\mathbf{d}_{h}: U \rightarrow \mathbb{R}^{d}: \mathbf{n} \mapsto \frac{1}{h(\mathbf{n})} \mathbf{n}
$$

Let $\mathbf{P}_{h}(\mathbf{n})$ be the tangent plane of the dual surface at the point $\mathbf{d}_{h}(\mathbf{n})$. This tangent plane is well defined for all $\mathbf{n} \in U$. By applying the polarity $\pi$ to the tangent planes, we obtain the primal surface associated with the function $h$, $\mathbf{p}_{h} \in \mathcal{C}^{\infty}\left(U, \mathbb{R}^{d}\right)$

$$
\mathbf{p}_{h}: \quad U \rightarrow \mathbb{R}^{d}: \quad \mathbf{n} \mapsto \mathbf{p}_{h}(\mathbf{n})=\pi\left(\mathbf{P}_{h}(\mathbf{n})\right)
$$

In addition, we may apply the polarity to the points of the dual surface,

$$
\mathbf{D}_{h}(\mathbf{n})=\pi\left(\mathbf{d}_{h}(\mathbf{n})\right)=\{\mathbf{x}: \mathbf{x} \cdot \mathbf{n}=h(\mathbf{n})\} .
$$

For each point $\mathbf{n} \in U \subseteq S$ we obtain a plane with the normal vector $\mathbf{n}$ and the oriented distance $h(\mathbf{n})$ to the origin. This plane is the tangent plane of the primal surface at $\mathbf{p}_{h}(\mathbf{n})$, provided that this point is regular. 
Consequently, the given function $h$ specifies the distance of the tangent planes of the dual surface to the origin. This function is called the support function of the primal surface.

The primal surface can be expressed directly with the help of the support function,

$$
\mathbf{p}_{h}(\mathbf{n})=h(\mathbf{n}) \mathbf{n}+\left(\nabla_{S} h\right)(\mathbf{n}) .
$$

The vector $\nabla_{S} h$ is obtained by embedding the intrinsic gradient of $h$ with respect to $S$ into the space $\mathbb{R}^{d}$. If the support function $h$ is the restriction of a function $h^{*} \in \mathcal{C}^{\infty}\left(\mathbb{R}^{d}, \mathbb{R}\right)$ to $U$, then it can be obtained by projecting the usual gradient into the tangent plane of the sphere,

$$
\left(\nabla_{S} h\right)(\mathbf{n})=\left(\nabla h^{*}\right)(\mathbf{n})-\left[\left(\nabla h^{*}\right)(\mathbf{n}) \cdot \mathbf{n}\right] \mathbf{n},
$$

see e.g. Š́r et al. (200x) for more information. $C^{\infty}$ parameterizations of the dual and the primal surfaces can be generated by composing (3) and (7) with suitable parameterizations of the spherical domain $U \subseteq S$.

The mapping $\mathbf{p}_{h} \in \mathcal{C}^{\infty}\left(U, \mathbb{R}^{d}\right)$ is the inverse Gauss map (see Kreyszig, 1991) of the primal surface, as its argument is simultaneously the unit normal vector of the tangent plane at the image point. Consequently, the curvature properties can be analyzed with the help of the differential of this mapping (see Šrir et al., 200x).

Hoschek (1983) uses dual surfaces associated with a given primal surface in order to detect parabolic points. He uses the polarity with respect to the "imaginary unit sphere".

\section{Rational support functions}

We introduce rational support functions and decompose them into symmetric and antisymmetric components. In addition we investigate some general properties of the associated surfaces.

\subsection{Surfaces with rational support functions}

Definition 1 A rational function $h$ on the unit sphere $S \subset \mathbb{R}^{d}$ is the restriction of a rational function $h^{*}$ on $\mathbb{R}^{d}$ to $S$.

This definition requires a slight clarification. Rational functions on $\mathbb{R}^{d}$ are not defined on the whole space, due to the zero set of the denominator. As common practice in algebraic geometry, we will simply ignore this fact, since 
the rational function are properly defined on a set which is open and dense in both Euclidean and Zarisky topology. This property is inherited by the restrictions to the sphere, provided that the denominator of $h^{*}$ does not contain the equation of the sphere as a factor.

By composing (3) and (7) with suitable rational parameterizations of the sphere one obtains the following result.

Proposition 1 The primal and dual surfaces associated with rational support functions admit rational parameterizations.

The class of primal surfaces associated with rational support functions are closed under translation, rotation, scaling, offsetting and convolution. This is due to the fact, that these geometrical operations are reflected by simple modifications of support functions, which preserve rationality.

More precisely the primal surface is rotated by a matrix $Q \in S O(d)$, i.e, $\mathbf{p}_{h} \mapsto Q \mathbf{p}_{h}$, then the new support function is $\mathbf{n} \mapsto h\left(Q^{-1} \mathbf{n}\right)$. If the surface is translated by a vector $\mathbf{a} \in \mathbb{R}^{d}$, i.e., $\mathbf{p}_{h} \mapsto \mathbf{p}_{h}+\mathbf{a}$, then the new support function is $\mathbf{n} \mapsto h(\mathbf{n})+\mathbf{a} \cdot \mathbf{n}$. Scaling corresponds to multiplying the support function by the scaling factor, and offsetting to adding the (oriented) offset distance. Finally the convolution of two primal surfaces corresponds to the addition of support functions ${ }^{1}$. This fact has been exploited by the authors in order to derive algorithms for computing the boundaries of Minkowski sums of planar and spatial domains (Š́r et al., 2007).

Remark 1 We use the notion of primal and dual surfaces merely in order to distinguish between the two surfaces (3) and (7) which are associated with a given support function. This notion does not imply additional properties of these surfaces; almost any surface can locally be considered either as a primal or dual surface associated with a certain support function.

\subsection{Even and odd functions}

Similar to rational functions on the real line, one may study certain symmetries. The notion of odd and even function is again inspired by properties of polynomials of odd and even degree.

Definition 2 A rational function $h$ on the sphere $S$ is said to be even resp. odd if $f(-\mathbf{n})=f(\mathbf{n})$ resp. $f(-\mathbf{n})=-f(\mathbf{n})$ holds for all $\mathbf{n} \in S$.

1 Here, the convolution of two surfaces consists of the sums of the coordinate vectors of all pairs of points where the associated tangent planes are parallel and the corresponding normal vectors point to the same side of the tangent planes. 
Moreover, any rational function on $S$ can be decomposed into a symmetric and an antisymmetric part.

Lemma 1 Any rational function $h$ on the sphere possesses a unique representation of the form

$$
h=h_{0}+h_{1},
$$

where $h_{0}$ is an even rational function and $h_{1}$ is an odd rational function.

Proof. The values of $h_{0}$ and $h_{1}$ are uniquely determined as

$$
h_{0}(\mathbf{n})=\frac{1}{2}(h(\mathbf{n})+h(-\mathbf{n})) \text { and } h_{1}(\mathbf{n})=\frac{1}{2}(h(\mathbf{n})-h(-\mathbf{n})) .
$$

Since $h$ is the restriction of a rational function $h^{*}$, the odd and even function are rational, since they are restrictions of the rational functions

$$
h_{0}^{*}(\mathbf{n})=\frac{1}{2}\left(h^{*}(\mathbf{n})+h^{*}(-\mathbf{n})\right) \text { and } h_{1}^{*}(\mathbf{n})=\frac{1}{2}\left(h^{*}(\mathbf{n})-h^{*}(-\mathbf{n})\right)
$$

to the sphere.

\subsection{The canonical extension}

A rational function on $S$ can be obtained by restricting various different rational functions on $\mathbb{R}^{d}$. The following results leads to a canonical extension.

Lemma 2 For any rational function $h$ on $S$ there is a unique rational extension $h^{+}$on $\mathbb{R}^{d}$ which is affine along the lines through the origin. More precisely, $h^{+}$satisfies

$$
h^{+}(\lambda \alpha \mathbf{n}+(1-\lambda) \beta \mathbf{n})=\lambda h^{+}(\alpha \mathbf{n})+(1-\lambda) h^{+}(\beta \mathbf{n})
$$

and

$$
h^{+}(\mathbf{n})=h(\mathbf{n})
$$

for all $\alpha, \beta, \lambda \in \mathbb{R}, \alpha, \beta \neq 0$ and $\mathbf{n} \in S$.

Proof. We prove this observation by constructing $h^{+}$from $h$. Since $h$ is rational, it is a restriction of a rational function

$$
h^{*}=\frac{p}{q}=\frac{p}{q_{0}+q_{1}}=\frac{p\left(q_{0}-q_{1}\right)}{\left(q_{0}+q_{1}\right)\left(q_{0}-q_{1}\right)}=\frac{p q_{0}-p q_{1}}{q_{0}^{2}-q_{1}^{2}}=\frac{r_{0}}{q_{0}^{2}-q_{1}^{2}}+\frac{r_{1}}{q_{0}^{2}-q_{1}^{2}}
$$

where $p, q, q_{0}, q_{1}, r_{0}, r_{1} \in \mathbb{R}\left[x_{1}, \ldots, x_{d}\right]$ are polynomials on $\mathbb{R}^{d}$. The polynomials $q_{0}$ and $q_{1}$ contain the terms of even and odd degree of $q=q_{0}+q_{1}$, respectively. Similarly, the polynomials $r_{0}$ and $r_{1}$ contain the terms of even and odd degree 
of $p\left(q_{0}-q_{1}\right)=r_{0}+r_{1}$, respectively. The denominator $s=q_{0}^{2}-q_{1}^{2}$ contains only terms of even degree.

By multiplying the terms of $s, r_{0}$ and $r_{1}$ with suitable powers of the homogeneous quadratic polynomial

$$
\|\mathbf{x}\|^{2}=x_{1}^{2}+\cdots+x_{d}^{2}
$$

which equals 1 when restricted to $S$, we obtain homogeneous polynomials $\hat{s}, \hat{r}_{0}$ of even degree and $\hat{r}_{1}$ of odd degree. When restricted to $S$, these polynomials coincide with $s, r_{0}$ and $r_{1}$.

Consider the rational function

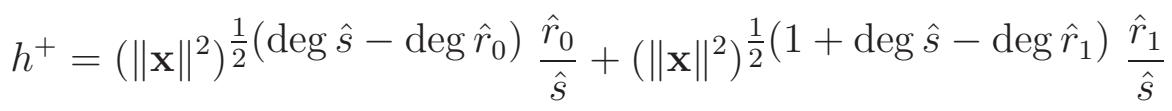

where $\operatorname{deg} p$ denotes the degree of $p$. The first term is constant along the lines through the origin, while the second term is homogeneous linear along these lines. Consequently, $h^{+}$is an affine function as defined in (11). Moreover (12) is also satisfied.

Finally, we note that $h^{+}$is unique, since the value $h^{+}(\mathbf{x})$ at $\mathbf{x} \in \mathbb{R}^{d} \backslash\{\mathbf{0}\}$ is determined by the values of $h$, as the identity

$$
\mathbf{x}=\frac{1-\|\mathbf{x}\|}{2}\left(-\frac{\mathbf{x}}{\|\mathbf{x}\|}\right)+\frac{1+\|\mathbf{x}\|}{2}\left(\frac{\mathbf{x}}{\|\mathbf{x}\|}\right)
$$

implies

$$
h^{+}(\mathbf{x})=\frac{1-\|\mathbf{x}\|}{2} h\left(-\frac{\mathbf{x}}{\|\mathbf{x}\|}\right)+\frac{1+\|\mathbf{x}\|}{2} h\left(\frac{\mathbf{x}}{\|\mathbf{x}\|}\right),
$$

due to (11) and (12).

Definition 3 The rational extension $h^{+} \in \mathcal{C}^{\infty}\left(\mathbb{R}^{d}, \mathbb{R}\right)$ from Lemma 2 will be called the canonical extension of the rational function $h \in \mathcal{C}^{\infty}(S, \mathbb{R})$ to the embedding space. From now on, if there is no danger of confusion, we will not distinguish between $h$ and $h^{+}$.

The mapping $h \rightarrow h^{+}$, which assigns the canonical extension to any (rational) function on $S$, is a linear operator which commutes with rotations around the origin. More precisely, for any rotation $\rho$ around the origin we have that $(\rho \circ h)^{+}=\rho \circ h^{+}$.

The simple expressions for the geometrical operations (translation, rotation, scaling offsetting, convolution) apply not only to the support functions, but also to the corresponding canonical extensions. 


\section{Surfaces with odd rational support functions}

We study certain geometric properties and discuss the relation to LN surfaces, in particular to quadratic triangular Bézier surfaces.

\subsection{Geometric properties}

This section is devoted to geometric properties of the primal and dual surfaces associated with odd rational support functions. The class of surfaces with odd rational support functions is closed under translation, rotation, scaling and convolution, but not offsetting.

In this case, the canonical extension defined in (11) is homogeneous linear along the lines through the origin, i.e., the first term in (15) is not present. Consequently the support function satisfies

$$
h(\lambda \mathbf{x})=\lambda h(\mathbf{x}), \quad \mathbf{x} \in \mathbb{R}^{d}, \quad \lambda \in \mathbb{R} .
$$

It follows that the gradient field $\nabla h$ is homogeneous of degree 0, i.e. constant along the lines through the origin,

$$
(\nabla h)(\lambda \mathbf{x})=(\nabla h)(\mathbf{x}), \quad \mathbf{x} \in \mathbb{R}^{d}, \quad \lambda \in \mathbb{R} \backslash\{0\} .
$$

The next theorem describes the main results concerning the associated primal and dual surfaces.

Theorem 3 The following three statements are equivalent.

(1) $h$ is an odd rational support function. More precisely, the rational function $h$ satisfies $h(\mathbf{n})=-h(-\mathbf{n})$.

(2) The primal surface associated with $h$ is an LN surface, i.e., it is a rational surface which can be equipped with a linear field of normal vectors.

(3) The dual surface associated with $h$ is a monoid surface with the singularity at the origin. More precisely, it is an algebraic surface which possesses a point of multiplicity $k-1$ at the origin, where $k$ is the algebraic order of the surface.

Remark 2 Sampoli et al. (2006) discuss LN surfaces in more detail. As observed there, they are dual to graphs of rational functions, which implies the equivalence $(2) \Leftrightarrow(3)$. Monoid surfaces have recently been studied by Johansen et al. (2007).

Proof. $(1) \Rightarrow(3)$ : The dual surface has the implicit equation

$$
h(\mathbf{x})-1=0
$$


where $h$ is the canonical extension of the odd rational support function. Indeed, the dual surface is given by (3), and using (18) we get

$$
h\left(\frac{\mathbf{n}}{h(\mathbf{n})}\right)=\frac{1}{h(\mathbf{n})} h(\mathbf{n})=1 .
$$

Since $h$ is an odd rational function, the canonical extension equals $h=p / q$, where $p, q$ are homogeneous polynomials of degree $m+1$ and $m$, respectively. Then the equation (20) can be written as

$$
p(\mathbf{x})-q(\mathbf{x})=0
$$

which represents a monoid surface with the singularity at the origin.

$(3) \Rightarrow(2)$ : Any monoid surface of order $m$ has an implicit representation of the form (22), where $p, q$ are homogeneous polynomials of degree $m+1$ and $m$, respectively. Let $h=p / q$. The vector field $\nabla h$ is constant along the lines through the origin and satisfies

$$
\mathbf{x} \cdot(\nabla h)(\mathbf{x})=h(\mathbf{x}), \quad \mathbf{x} \in \mathbb{R}
$$

In order to find the primal surface, we apply the polarity to the dual surface defined by (22) or equivalently by (20). For any $\mathbf{n} \in S$, the line $\mathbb{R} \mathbf{n}$ intersects the surface in the origin and in another point $\mathbf{n} / h(\mathbf{n})$. A normal of the tangent plane at this point can be obtained as the gradient of the left-hand side of Eq. (20),

$$
(\nabla h)\left(\frac{\mathbf{n}}{h(\mathbf{n})}\right)=(\nabla h)(\mathbf{n}) .
$$

The tangent plane has the equation

$$
\mathbf{x} \cdot(\nabla h)(\mathbf{n})=\frac{\mathbf{n}}{h(\mathbf{n})} \cdot(\nabla h)(\mathbf{n})=1
$$

where we used (23) in order to simplify the right-hand side. Consequently, the polarity $\pi$ maps the tangent plane of the monoid surface to the point $(\nabla h)(\mathbf{n})$ of the primal surface, and $\mathbf{n}$ is the unit normal at this point. Now, by composing the central projection $\mathbf{x} \rightarrow \mathbf{x} /\|\mathbf{x}\|$ onto the sphere with the parameterization $\mathbf{n} \rightarrow(\nabla h)(\mathbf{n})$ of the primal surface, we obtain the rational mapping

$$
\mathbb{R}^{d} \backslash\{\mathbf{0}\} \rightarrow \mathbb{R}^{d}: \quad \mathbf{x} \rightarrow(\nabla h)\left(\frac{\mathbf{x}}{\|\mathbf{x}\|}\right)=(\nabla h)(\mathbf{x})
$$

which maps any point $\mathbf{x}$ to a point of the primal surface, such that $\mathbf{x}$ is a normal to the surface at this point. Once more we exploited the observation that $\nabla h$ is constant along lines through the origin. By restricting this mapping to a linear parameterization of an arbitrary plane not passing through the origin we obtain an LN parameterization of the primal surface. 
$(2) \Rightarrow(1)$ : A rational LN-surface is described by a rational parameterization $\mathbf{r}\left(u_{1}, \ldots, u_{d-1}\right)$ and it is equipped with a linear field of (non-unit) normal vectors. After a suitable reparameterization and choice of coordinates, the field of normal vectors takes the form $\left(1, u_{1}, \ldots, u_{d-1}\right)^{\top}$, see Sampoli et al. (2006). Consequently, the unit normals are

$$
\mathbf{n}\left(u_{1}, \ldots, u_{d-1}\right)=\left(n_{1}, \ldots, n_{d}\right)^{\top}=\left(\frac{1}{\sqrt{Q}}, \frac{u_{1}}{\sqrt{Q}}, \ldots, \frac{u_{d-1}}{\sqrt{Q}}\right)^{\top},
$$

where $Q=1+u_{1}^{2}+\cdots+u_{d-1}^{2}$, which implies the relations

$$
u_{i}=\frac{n_{i+1}}{n_{1}}, \quad i=1, \ldots, d-1, \text { and } n_{1}=\frac{1}{\sqrt{Q}}
$$

between surface parameters and components of the unit normal (27). Using these relations, the distance of the tangent plane at a point $\mathbf{r}\left(u_{1}, \ldots, u_{d-1}\right)$ to the origin evaluates to

$$
\begin{aligned}
h\left(n_{1}, \ldots, n_{d}\right) & =\mathbf{r}\left(u_{1}, \ldots, u_{d-1}\right) \cdot \mathbf{n}\left(u_{1}, \ldots, u_{d-1}\right) \\
& =n_{1} \mathbf{r}\left(\frac{n_{2}}{n_{1}}, \ldots, \frac{n_{d}}{n_{1}}\right) \cdot\left(1, \frac{n_{2}}{n_{1}}, \ldots, \frac{n_{d}}{n_{1}}\right)^{\top} .
\end{aligned}
$$

Obviously this is an odd rational function on the unit sphere.

\subsection{LN surfaces and convolutions}

As observed in the second part of the proof, the points of the primal surface are obtained as

$$
\mathbf{p}_{h}(\mathbf{n})=(\nabla h)(\mathbf{n})
$$

where $h$ is the canonical extension of the odd rational support function. Points that are associated with opposite normals are identical,

$$
\nabla h(\mathbf{n})=\nabla h(-\mathbf{n}),
$$

as the gradient field $\nabla h$ is constant along the lines through the origin. If we compute an offset surface at distance $d$, then the points associated with opposite normals are

$$
\mathbf{p}_{h+d}(\mathbf{n})=(\nabla h)(\mathbf{n})+\mathbf{n} d \quad \text { and } \quad \mathbf{p}_{h+d}(-\mathbf{n})=(\nabla h)(-\mathbf{n})-\mathbf{n} d,
$$

i.e., they have the constant distance $2 d$. This proves the following corollary:

Theorem 4 The offsets of LN surfaces are surfaces of constant width.

Curves and surfaces of constant width have been studied extensively in convex geometry, see e.g. (Strubecker, 1959; Guilfoyle and Klingenberg, 2007). 

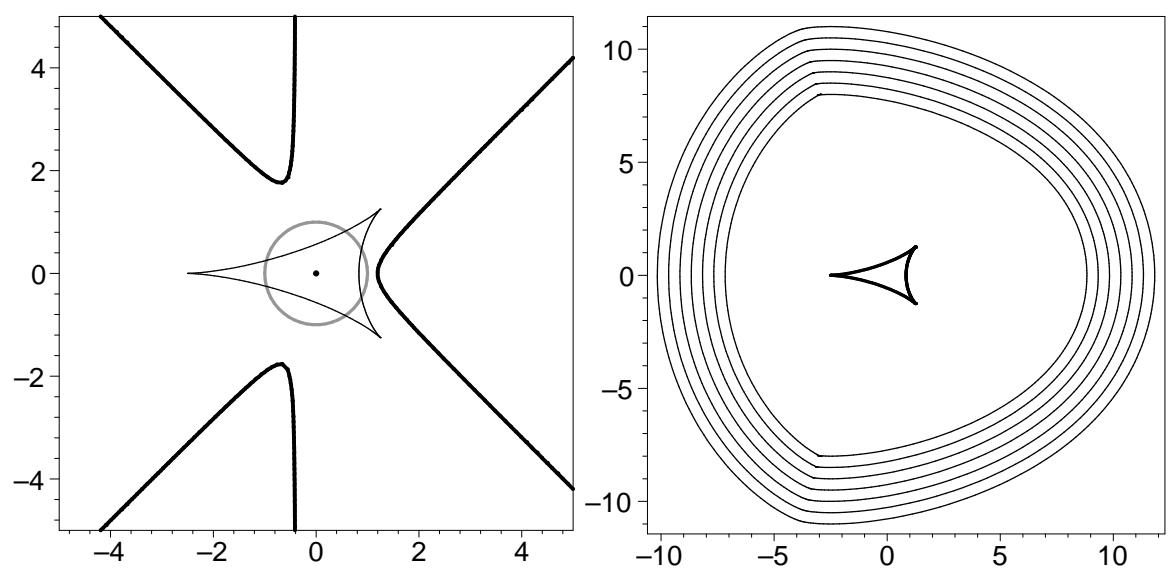

Fig. 1. Left: A curve with odd rational support function and its dual curve (thick). Right: The offsets are curves of constant width.

Example 1 We consider the curve $(d=2)$ with the odd rational support function

$$
h=\frac{5}{2} \frac{x^{3}-x y^{2}}{3 x^{2}+y^{2}} .
$$

The dual curve is a monoid of degree 3, which has an isolated double point at the origin. The curve and its dual curve and some offsets are shown in Fig. 1. For smaller values of the offset distance, the offsets have self-intersections.

The next result follows directly from (19) and (30). It provides an alternative approach to the rational convolution property of LN surfaces, which was also observed by Sampoli et al. (2006).

Corollary 5 Let $d=3$. Consider a surface $\mathbf{x}\left(u_{1}, u_{2}\right)$ with parameters $\left(u_{1}, u_{2}\right) \in$ $\Omega \subseteq \mathbb{R}^{2}$, along with a surface which is described by an odd rational support function. The convolution of both surfaces can be obtained as

$$
\mathbf{x}\left(u_{1}, u_{2}\right)+(\nabla h)\left(\mathbf{x}_{1}\left(u_{1}, u_{2}\right) \times \mathbf{x}_{2}\left(u_{1}, u_{2}\right)\right)
$$

where $\mathbf{x}_{i}$ denotes the partial derivative vector with respect to $u_{i}$. If $\mathbf{x}$ is a rational surface, then the convolution surface is rational, too.

\subsection{Quadratic triangular Bézier surfaces}

As shown recently, quadratic triangular Bézier surfaces ${ }^{2}$ (except for developable ones) belong to the class of LN surfaces. Lávička and Bastl (2007) proved this result in a more general framework using Gröbner bases, while Peternell and Odehnal (2007) presented a different approach which relies on the

2 A general classification of these surfaces has been given by Degen (1996) and Peters and Reif (1998). 
analysis of Cremona transformations. We present an example which demonstrates that these surfaces possess odd rational support functions, which can be expressed as the quotient of a cubic and a quadratic homogeneous polynomial. It is shown that the support functions can be found simply by solving a $2 \times 2$ system of linear equations. The approach can be extended to hypersurfaces with quadratic parameterizations in $\mathbb{R}^{d}$.

Example 2 We consider a quadratic triangular Bézier surface patch in $\mathbb{R}^{3}$,

$$
\mathbf{x}(u, v, w)=\sum_{\substack{i, j, k \in \mathbb{Z}_{+} \\ i+j+k=2}} \frac{2}{i ! j ! k !} u^{i} v^{j} w^{k} \mathbf{b}_{i, j, k}, \quad u+v+w=1 .
$$

The support function $h$ can be found by eliminating $u, v$ from the following system of equations:

$$
\begin{gathered}
h=\mathbf{n} \cdot \mathbf{x}(u, v, 1-u-v), \\
0=\mathbf{n} \cdot \frac{\partial}{\partial u} \mathbf{x}(u, v, 1-u-v), \quad 0=\mathbf{n} \cdot \frac{\partial}{\partial v} \mathbf{x}(u, v, 1-u-v) .
\end{gathered}
$$

The last two equations are linear in $u, v$. They are used to express these parameters in terms of $\mathbf{n}=\left(n_{1}, n_{2}, n_{3}\right)$. By substituting the result into the first equation one finds the support function of the surface. For instance, if we consider a surface patch with the control points

$$
\begin{gathered}
\mathbf{b}_{2,0,0}=(1,0,0)^{\top}, \mathbf{b}_{0,2,0}=(0,1,0)^{\top}, \mathbf{b}_{0,0,2}=(0,0,1)^{\top}, \\
\mathbf{b}_{1,1,0}=\mathbf{b}_{1,0,1}=\mathbf{b}_{0,1,1}=(0,0,0)^{\top} .
\end{gathered}
$$

then the support function takes the form

$$
h\left(n_{1}, n_{2}, n_{3}\right)=\frac{n_{1} n_{2} n_{3}}{n_{1} n_{2}+n_{1} n_{3}+n_{2} n_{3}} .
$$

\section{Surfaces with even rational support functions}

We study geometric properties of the primal and dual surfaces associated with even rational support functions. The class of surfaces with even rational support functions is closed under rotation, scaling, offsetting and convolution, but not translation ${ }^{3}$.

In this case, the canonical extension defined in (11) is constant along the lines through the origin, i.e., the second term in (15) is not present. Consequently

$\overline{3}$ A translation-invariant class of surfaces can be obtained by considering support functions that are sums of even rational functions and linear polynomials. 
the support function satisfies

$$
h(\lambda \mathbf{x})=h(\mathbf{x}), \quad \mathbf{x} \in \mathbb{R}^{d}, \quad \lambda \in \mathbb{R} \backslash\{0\}
$$

The next theorem describes the main results concerning the associated primal and dual surfaces.

Theorem 6 The following three statements are equivalent.

(1) $h$ is an even rational support function. More precisely, the rational function $h$ satisfies $h(\mathbf{n})=h(-\mathbf{n})$.

(2) The primal surface associated with $h$ possesses a non-rational parameterization of the form

$$
\left(u_{1}, \ldots, u_{d-1}\right) \rightarrow \pm \frac{1}{\sqrt{Q}} \mathbf{r}\left(u_{1}, \ldots, u_{d-1}\right),
$$

such that the vectors $\left(1, u_{1}, \ldots, u_{d-1}\right)^{\top}$ form a field of normal vectors, where $\mathbf{r} \in \mathcal{C}^{\infty}\left(\mathbb{R}^{d-1}, \mathbb{R}^{d}\right)$ is a rational function and $Q=1+u_{1}^{2}+\cdots+u_{d-1}^{2}$. Since the normal vectors depend linearly on the surface we call (40) an LN parameterization of the surface.

(3) The dual surface associated with $h$ has the following properties:

(i) It is a surface of a certain even degree $2 m+2$ which is centrally symmetric with respect to the origin.

(ii) It has a point of multiplicity $2 m$ at the origin.

(iii) The tangent cone at the origin is a "double cone" of order $m$.

(iv) Its intersection with the plane at infinity factorizes as the absolute circle (sphere) and a double $(d-2)$-dimensional surface.

Proof. $(1) \Rightarrow(3)$ : The dual surface has the implicit equation

$$
h(\mathbf{x})^{2}\|\mathbf{x}\|^{2}-1=0
$$

where $h$ is the canonical extension of the even rational support function. Indeed, the dual surface is given by (3), and using (39) we get

$$
h^{2}\left(\frac{\mathbf{n}}{h(\mathbf{n})}\right)\left\|\frac{\mathbf{n}}{h(\mathbf{n})}\right\|^{2}=h^{2}(\mathbf{n}) \frac{\|\mathbf{n}\|^{2}}{h^{2}(\mathbf{n})}=\|\mathbf{n}\|^{2}=1 .
$$

and it is easy to see that all points satisfying (41) belong to the dual surface.

The canonical extension of the support function takes the form $h=p / q$, where $p, q$ are two homogeneous polynomials on $\mathbb{R}^{d}$ of the same degree $m$. Eq. (41) can therefore be rewritten as

$$
p(\mathbf{x})^{2}\|\mathbf{x}\|^{2}-q(\mathbf{x})^{2}=0
$$


The class of algebraic surfaces of this form can equivalently be characterized by the four geometric conditions (i)-(iv). First, Eq. (43) has degree $2 m-$ 2 and it contains only terms of even degree, hence the surface is centrally symmetric with respect to the origin. Second, the first non-vanishing term of the Taylor expansion at the origin is $q^{2}$, hence we have (ii) and (iii). Finally, the intersection with the plane at infinity is $p(\mathbf{x})^{2}\|\mathbf{x}\|^{2}=0$, which is characterized by (iv).

$(3) \Rightarrow(2)$ : We assume that the dual surface is given by an equation of the form (43), where $p, q$ are homogeneous polynomials of degree $m$. Let $h=p / q$. This function is homogeneous rational of degree 0 (characterized by (39)), and the gradient field $\nabla h$ is homogeneous rational of degree -1 ,

$$
(\nabla h)(\lambda \mathbf{x})=\frac{1}{\lambda}(\nabla h)(\mathbf{x}), \quad \lambda \neq 0 .
$$

Moreover, since $h$ is constant along the lines through the origin,

$$
\mathbf{x} \cdot \nabla h(\mathbf{x})=0
$$

holds for all $\mathbf{x} \neq \mathbf{0}$. We compute the image of the dual surface under the polarity $\pi$. Any line $\mathbb{R} \mathbf{n}$ intersects the surface in the origin and in two additional points

$$
\frac{1}{h(\mathbf{n})} \mathbf{n} \quad \text { and } \quad-\frac{1}{h(\mathbf{n})} \mathbf{n}=\frac{1}{h(-\mathbf{n})}(-\mathbf{n})
$$

The normal vector of the dual surface is obtained as the gradient of (41),

$$
\nabla\left(h(\mathbf{x})^{2}\|\mathbf{x}\|^{2}-1\right)=2 h(\mathbf{x})(\nabla h)(\mathbf{x})\|\mathbf{x}\|^{2}+2 \mathbf{x} h(\mathbf{x})^{2} .
$$

Setting $\mathbf{x}=(1 / h(\mathbf{n})) \mathbf{n}$ we obtain a normal at this point. With the help of (39) and (44), this can be simplified to

$$
2[(\nabla h)(\mathbf{n})+h(\mathbf{n}) \mathbf{n}] .
$$

The tangent plane at the first intersection point possesses the equation

$$
\mathbf{x} \cdot[\nabla h(\mathbf{n})+h(\mathbf{n}) \mathbf{n}]=\frac{1}{h(\mathbf{n})} \mathbf{n} \cdot[(\nabla h)(\mathbf{n})+h(\mathbf{n}) \mathbf{n}]=1,
$$

where we used (45) in order to eliminate the first term on the right-hand side. Similarly we obtain the tangent plane

$$
\mathbf{x} \cdot(\nabla h(-\mathbf{n})+h(-\mathbf{n})(-\mathbf{n}))=1
$$

at the second intersection point. Thus, for any $\mathbf{n} \in S$, the point $(\nabla h)(\mathbf{n})+$ $h(\mathbf{n}) \mathbf{n}$ belongs to the the primal surface and $\mathbf{n}$ is a unit normal at this point. Now, composing the mapping $\mathbf{x} \rightarrow \frac{\mathbf{x}}{\|\mathbf{x}\|}$ with the parameterization

$$
\mathbf{n} \rightarrow(\nabla h)(\mathbf{n})+h(\mathbf{n}) \mathbf{n}
$$




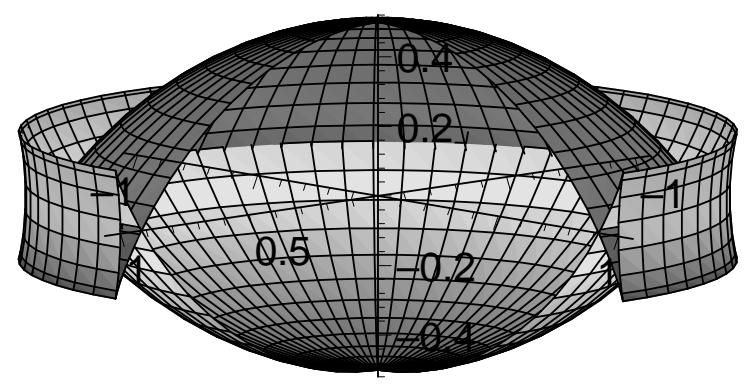

$$
h=\frac{1}{1+z^{2}} \quad h=\frac{x^{2}+2 y^{2}}{1+z^{2}}
$$

Fig. 2. Primal surfaces associated with even rational support functions.

of the primal surface, we obtain the mapping

$$
\mathbf{x} \rightarrow \frac{1}{\|\mathbf{x}\|}\left(\|\mathbf{x}\|^{2}(\nabla h)(\mathbf{x})+h(\mathbf{x}) \mathbf{x}\right)
$$

from $\mathbb{R}^{d}-\{0\}$ to the primal surface, such that $\mathbf{x}$ is a normal to the surface at the corresponding point. Restricting this mapping to the planes $x_{1}= \pm 1$, which can be parameterized as $\mathbf{x}= \pm\left(1, u_{1}, \ldots, u_{d-1}\right)$, we obtain the parameterizations (40).

$(2) \Rightarrow(1)$ : First we define an orientation of the two branches of the surface (40): The vectors

$$
\pm \frac{1}{\sqrt{Q}}\left(1, u_{1}, \ldots, u_{d-1}\right)^{\top}
$$

are unit normals of the corresponding branch (with the same sign). Consequently, the unit vector $\mathbf{n}=\left(n_{1}, \ldots, n_{d}\right)$ is normal vector at the point obtained by choosing $u_{i}=n_{i+1} / n_{1}$, where the sign of $n_{1}$ specifies the branch. This gives

$$
\operatorname{sgn}\left(n_{1}\right)\left(\sqrt{1+\frac{n_{2}^{2}}{n_{1}^{2}}+\ldots+\frac{n_{d}^{2}}{n_{1}^{2}}}\right)^{-1} \mathbf{r}\left(\frac{n_{2}}{n_{1}}, \ldots, \frac{n_{d}}{n_{1}}\right)=n_{1} \mathbf{r}\left(\frac{n_{2}}{n_{1}}, \ldots, \frac{n_{d}}{n_{1}}\right)
$$

Hence, the support function

$$
h\left(n_{1}, \ldots n_{d}\right)=n_{1} \mathbf{r}\left(\frac{n_{2}}{n_{1}}, \ldots, \frac{n_{d}}{n_{1}}\right) \cdot \mathbf{n}
$$

is an even rational function. Choosing the opposite orientation of the surface changes the sign of the support function.

Example 3 Fig. 2 shows two examples of rational surfaces with even rational support functions. Note that 1 in the equations can be replaced with $\|\mathbf{x}\|^{2}=$ $x^{2}+y^{2}+z^{2}$, in order to obtain the canonical extension of $h$ to the embedding space. 
Example 4 We consider the primal and the dual surfaces which are defined by the support function $h(x, y, z)=\frac{x}{z}$ and analyze them in more detail. According to the proof of Theorem 6 , the associated dual surface has the implicit equation

$$
x^{2}\left(x^{2}+y^{2}+z^{2}\right)-z^{2}=0 .
$$

This algebraic surface of degree 4 surface possesses two double lines: the line $x=z=0$ and intersection of the plane $x=0$ with the plane at infinity. These two double lines intersect in a point of multiplicity 2, which is located at the infinite point of the $y$ axis. The origin is a double point with the tangent cone $z^{2}=0$, which is a double plane. The dual surface is a Del Pezzo surface of degree 4 (see Schicho, 2005). The dual and primal surfaces are shown in Fig. 3.
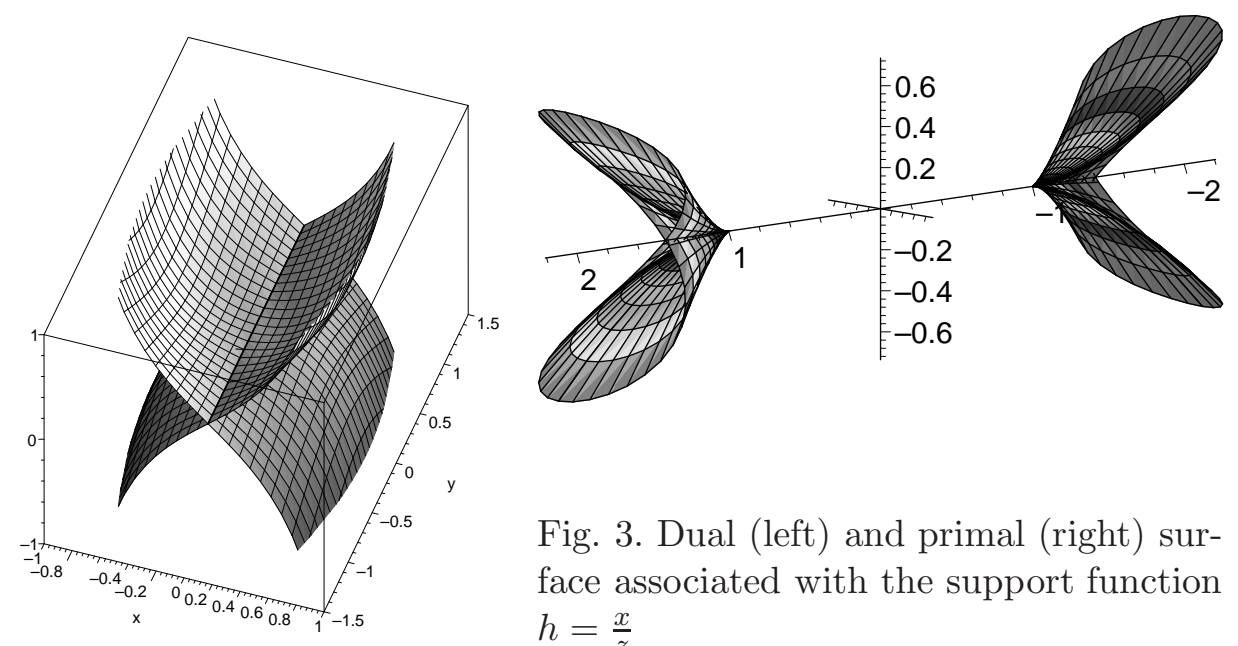

Fig. 3. Dual (left) and primal (right) surface associated with the support function $h=\frac{x}{z}$

\section{Conclusion}

Surfaces with a rational support function can be obtained as the convolution of two surfaces with an odd and an even rational support function, respectively. We analyzed the relations of the odd components to the class of LN surfaces, which was studied by Sampoli et al. (2006). In addition, the geometry of the components was characterized by geometric properties of the dual surfaces.

As a major limitation, surfaces defined by support functions cannot represent parabolic points, as those points correspond to singularities of the Gauss map. As a matter of future work we will explore multi-valued functions on the sphere, in order to deal with the representation of surfaces containing parabolic lines. 


\section{References}

P. Alfeld, M. Neamtu and L.L. Schumaker (1996), Fitting scattered data on spherelike surfaces using spherical splines, J. Comput. Appl. Math. 73, 5-43.

H. Almegaard, A. Bagger, J. Gravesen, B. Jüttler, and Z. Šír (2007), Surfaces with Piecewise Linear Support Functions over Spherical Triangulations, The Mathematics of Surfaces XII (R. Martin, M. Sabin and J. Winkler eds.), Springer LNCS vol. $4647,42-63$.

T. Bonnesen and W. Fenchel (1987): Theory of convex bodies. BCS Associates, Moscow, Idaho.

W.L.F Degen (1996): The types of quadratic triangular Bézier surfaces. In: The Mathematics of Surfaces VI (G. Mullineux, editor), Oxford University Press, 153-170.

R. Farouki and T. Sakkalis (1990): Pythagorean hodographs. IBM J. Res. Develop. 34, 736-752.

R. Farouki (2002): Pythagorean-hodograph curves, Handbook of Computer Aided Geometric Design, G. Farin, J. Hoschek, and M-S. Kim, eds., North Holland, 405-427.

J. Gravesen (2007): Surfaces parametrised by the normals, Computing 79, 175-183.

H. Groemer (1996): Geometric Applications of Fourier Series and Spherical Harmonics, Cambridge University Press, Cambridge, 1996.

P. M. Gruber and J. M. Wills, J. M. (1993): Handbook of convex geometry, NorthHolland, Amsterdam.

B. Guilfoyle and W. Klingenberg (2007): On $C^{2}$-smooth Surfaces of Constant Width, http://www.citebase.org/ abstract?id=oai:arXiv.org:0704.3248.

J. Hoschek (1983): Dual Bézier curves and surfaces, in: Surfaces in Computer Aided Geometric Design (R.E. Barnhill and W. Boehm, eds.), North Holland, 147-156.

H. Johansen, M. Løberg and R. Piene (2007): Monoid hypersurfaces. In: Geometric Modeling and Algebraic Geometry (R. Piene and B. Jüttler, eds.), Springer, to appear.

M. Lávička and B. Bastl (2007): Rational Hypersurfaces with Rational Convolutions. Comput. Aided Geom. Design 24, 410-426.

E. Kreyszig (1991): Differential Geometry. Dover, New York.

M. Peternell and B. Odehnal (2007): Convolution surfaces of quadratic triangular Bézier surfaces. Comput. Aided Geom. Design, in press.

M. Peternell and H. Pottmann (1998): A Laguerre geometric approach to rational offsets, Comput. Aided Geom. Design 15, 223-249.

J. Peters and I. Reif U (1998): The 42 equivalence classes of quadratic surfaces in affine n-space. Comput. Aided Geom. Design 15, 459-73.

H. Pottmann (1995), Rational curves and surfaces with rational offsets, Comput. Aided Geom. Design 12, 175-192.

M. Sabin (1974), A Class of Surfaces Closed under Five Important Geometric Operations, Technical report no. VTO/MS/207, British aircraft corporation, available at http://www.damtp.cam.ac.uk/user/na/people/ Malcolm/vtoms/vtos.html

M.L. Sampoli, M. Peternell and B. Jüttler (2006): Rational surfaces with linear normals and their convolutions with rational surfaces. Comput. Aided Geom. 
Design 23, 179-192.

J. Schicho (2005), Elementary Theory of del Pezzo Surfaces. In: Computational Methods for Algebraic Spline Surfaces (T. Dokken and B. Jüttler, eds.), Springer, 77-94.

Z. Šír, J. Gravesen and B. Jüttler (2007): Computing Minkowski sums via Support Function Representation, in Curve and Surface Design: Avignon 2006, (P. Chenin, T. Lyche L. Schumaker eds.) Nashboro Press, Brentwood, 244-253.

Z. Š́r, J. Gravesen, and B. Jüttler (200x), Curves and surfaces represented by polynomial support functions, Theoretical Computer Science, in press.

E. Strubecker (1959), Differentialgeometrie I-III. Sammlung Göschen. 